discussion at a time when peer support methods were arguably needed more than ever.

Objectives To pilot a facilitated peer support session in a virtual format and assess the acceptability of the format for trainees.

Methods A themed discussion entitled 'In This Bleak Midwinter' was incorporated into the January 2021 regional ST3 trainee study day, which was convened virtually via Zoom videoconferencing software. 21 ST3 trainees were split into three virtual breakout rooms, with at least one trainee per group given a short briefing beforehand and asked to prepare something to begin the discussion. One trainee was removed from the session due to camera issues, as the faculty felt video was crucial for maximising engagement with the session and maintaining the trust necessary to develop a 'safe space' for open discussion. Three members of a local chaplaincy team, trained in Schwartz and Balint methodology, facilitated group discussions which lasted for approximately 55 minutes. A scheduled break followed to allow trainees to reflect and recover before continuing with the rest of the day's programme. Feedback was gathered via anonymous online survey.

Results $85 \%$ of trainees rated the session as 'excellent' on a five-point Likert scale, the most positive rating possible. 58\% of respondents specifically mentioned the session in a free text question asking for 'three good things about the day'. Three trainees stated in a free text question asking 'how could the day be improved?' that they would like facilitated peer support sessions to be scheduled during every monthly teaching programme. One trainee subsequently sought professional help for their mental health and directly cited the session as the driver to do so.

Conclusions The considerable positive feedback suggests that facilitated peer support sessions can be successful when held in a virtual format. Data on lasting effects were not gathered and future research could try to ascertain whether the positive reactions produced medium-term and long-term benefits, similar to face-to-face sessions. Future research could also examine the effect of cameras on engagement, as this seemed the main barrier to participation and engagement in our pilot session.

\section{PAEDIATRIC LESS THAN FULL TIME TRAINING SURVEY ACROSS OUR REGION: AN INITIATIVE TO SUPPORT TRAINEES}

${ }^{1}$ Nithya Reddy, ${ }^{1}$ Francesca Seregni, ${ }^{2}$ Anshoo Dhelaria. ${ }^{1}$ Cambridge University Hospitals NHS Trust, ${ }^{2}$ East and North Hertfordshire NHS Trust

\subsection{6/archdischild-2021-rcpch.630}

Background Paediatrics has a higher number of Less Than Full Time (LTFT) trainees in comparison to other hospital specialities. $34 \%$ of paediatric trainees in our region are in less than full time training. Trainees working LTFT have a different work experience and can encounter different challenges. The paediatric LTFT trainee representatives and the consultant trainee programme director (TPD) for LTFT training organised a survey looking at different initiatives to support less than full time trainees.

Objectives Aim of the survey was to explore the experiences of the paediatric LTFT trainees across our deanery and to look at the measures to be put in place to improve.

Methods An anonymous questionnaire was created using survey monkey. This was distributed via email to all 86
LTFT trainees in our region and it was kept open for 4 weeks. Data was subsequently collated using Microsoft excel.

Results

- The questionnaire was completed by a total of 50 trainees $(58 \%)$ at all levels of training.

- Majority of the trainees opted to work $60 \%$ of the whole time equivalent slot.

- Childcare was the main reason for working LTFT (76\%).

- Most of the trainees (87\%) were able to get all 'Work Based Assessments (WBA)' required for the given training period.

- Only 55\% of the LTFT trainees received their Rota on time.

Conclusions

- Providing the Rota on time has been one of the major concerns across many trusts. At deanery level, we are now introducing a recommended timeline for rota production to facilitate the LTFT rota provision on time.

- In the following placements after the survey, LTFT trainees received the confirmation of their placements and their job sharers well in advance.

- We are aiming to conduct a LTFT survey annually to address trainees concerns.

\section{Paediatricians with Expertise in Cardiology Special Interest Group}

\section{ARTERIAL FUNCTION IN PREADOLESCENT CHILDREN WITH CONGENITAL HEART DISEASE: A SYSTEMATIC REVIEW}

${ }^{1}$ Ioanna Zimianiti, ${ }^{2}$ Chloe Cheang, ${ }^{3}$ Roshni Mansfield, ${ }^{1}$ Carmen Traseira Pedraz,

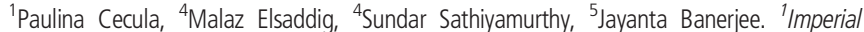
College London School of Medicine; ' Queen Charlotte's and Chelsea Hospital, Imperial College Healthcare NHS Trust; ${ }^{3}$ Department of Neonatology, Queen Charlotte's and Chelsea Hospital, Imperial College Healthcare NHS Trust; Biomedical Research Centre, Imperial College London; ${ }^{4}$ Department of Neonatology, Queen Charlotte's and Chelsea Hospital, Imperial College Healthcare NHS Trust; ${ }^{5}$ Department of Neonatology, Queen Charlotte's and Chelsea Hospital, Imperial College Healthcare NHS Trust; Biomedical Research Centre, Imperial College London; Institute of Reproductive and Developmental Biology, Imperial College London; Origins of Child Health and Disease, Centre for Paediatrics and Child Health, Imperial College London

\subsection{6/archdischild-2021-repch.63}

Background Congenital heart disease (CHD) can increase longterm cardiovascular disease risk. Studying arterial stiffness, an independent predictor of cardiovascular morbidity and mortality, can improve understanding of the pathophysiology of cardiovascular disease in CHD.

Objectives To systematically review the literature to examine how CHD affects arterial stiffness in children $\leq 12$ years, following PRISMA guidelines.

Methods PubMed was searched using the terms: 'pulse wave velocity' (PWV), 'carotid intima-media thickness' (cIMT), 'arterial stiffness index' (SIx), 'flow-mediated dilation' (FMD), 'flow imaging', 'laser flow Doppler', 'venous plethysmography', 'cardi" magnetic resonance imaging', 'aortic intima-media thickness' (aIMT), 'vascular ultrasound' and 'neonat"', 'paediatric', 'infant"', 'child"'. Case reports, case series, reviews, commentaries, conference proceedings, animal studies, articles not in English and articles with children $>12$ years were 